$12-1-1978$

\title{
Role of Low-Energy Two-Body Virtual States in Three-Particle Scattering Situations
}

James A. Lock

Cleveland State University, j.lock@csuohio.edu

Follow this and additional works at: https://engagedscholarship.csuohio.edu/sciphysics_facpub

Part of the Physics Commons

How does access to this work benefit you? Let us know!

Publisher's Statement

Copyright 1978 American Physical Society. Available on publisher's site at http://link.aps.org/ doi/10.1103/PhysRevC.18.2465.

\section{Original Citation}

Lock, James A. "Role of Low-Energy Two-Body Virtual States in Three-Particle Scattering Situations."

Physical Review C 18 (1978): 2465-2469.

\section{Repository Citation}

Lock, James A., "Role of Low-Energy Two-Body Virtual States in Three-Particle Scattering Situations" (1978).

Physics Faculty Publications. 20.

https://engagedscholarship.csuohio.edu/sciphysics_facpub/20

This Article is brought to you for free and open access by the Physics Department at EngagedScholarship@CSU. It has been accepted for inclusion in Physics Faculty Publications by an authorized administrator of

EngagedScholarship@CSU. For more information, please contact library.es@csuohio.edu. 


\title{
Role of low-energy two-body virtual states in three-particle scattering situations
}

\author{
J. A. Lock \\ Physics Department, Case Western Reserve University, Cleveland, Ohio 44106 \\ and Physics Department, Cleveland State University, Cleveland, Ohio 44115* \\ (Received 31 July 1978)
}

\begin{abstract}
A model three-particle scattering situation consisting of a projectile incident on a target bound to a residual core was considered under the assumptions that there was a low-energy virtual state in the projectile-target channel, that the projectile and core did not interact, and that the projectile-target and target-core interactions were $s$-wave separable interactions. When the appropriate Faddeev equations were solved by inversion, an enhancement was found in the three-body elastic cross section for virtual state energies less than or comparable to the target-core binding energy. This enhancement was determined to be kinematical in origin.
\end{abstract}

[NUCLEAR REACTIONS Three-body scattering theory, subsystem virtual] states. Enhancements in three-body elastic cross section.

For the three-body problem in the nonrelativistic Faddeev formalism, the input for the equations consists of the off-shell two-body subsystem $t$ matrices

$$
\left\langle\overrightarrow{\mathrm{k}}_{i j}^{\prime}\left|t_{i j}(E)\right| \overrightarrow{\mathrm{k}}_{i j}\right\rangle
$$

associated with the subsystem potentials $V_{i j}(E)$ where $\overrightarrow{\mathrm{k}}_{i j}^{\prime}$ and $\overrightarrow{\mathrm{k}}_{i j}$ are the initial and final relative momentum of the $i j$ pair and $E$ is the subsystem parametric energy. The poles of $t_{i j}$ as a function of $E$ are of three types: poles on the negative real axis of the physical $E$ sheet corresponding to bound states, poles on the negative real axis of the unphysical $E$ sheet corresponding to virtual states, and poles for complex values of $E$ on the unphysical $E$ sheet corresponding to scattering resonances for $\operatorname{Re}(E)>0, \operatorname{Im}(E) \lessgtr 0$. In the past, many studies have been undertaken to determine when and how various two-body subsystem dynamics, for instance bound states or resonances, influence or dominate the scattering in various threebody situations. However, almost nothing has been said concerning the possible influence of twobody virtual states in a model three-body problem. This omission may well be because of the apparent lack of two-body systems possessing a low lying virtual state. In the zero range approximation, the $s$-wave scattering amplitude possesses such a virtual state pole for negative scattering lengths. In the best known example of this, singlet $-S$ nucleon-nucleon scattering, the virtual state energy is roughly $65 \mathrm{keV}$. In comparison with the atomic binding of hydrogen atoms into molecules, this virtual state energy is very high obscuring any evidence of it in neutron-molecule scattering. In fact it is the relative largeness of this energy and the corresponding relative slow variation of the ${ }^{1} S_{0} n-p t$ matrix which allows Fermi's pseudopotential method ${ }^{1}$ to describe low-energy neutron- bound proton scattering so well.

It is the purpose of the present study to investigate the influence of a two-body subsystem virtual state on three-body scattering amplitudes. In particular, we choose the situation mentioned above of a neutron scattering from a proton bound to a hydrocarbon molecule under the assumptions that the neutron and "residual molecular core" do not interact, all particles are spinless, and that the $n-p$ and proton-core interactions may be reasonably represented by single term $s$-wave Yamaguchi separable potentials. This system was examined extensively in a previous paper. ${ }^{2}$ Just as resonances of a sufficiently narrow width generate a rapidly varying structure in the threebody scattering amplitude, so too as we artificially decrease the virtual state energy or equivalently increase the $n-p$ scattering length toward values comparable with those of proton-molecule binding, we may determine which virtual state energies are small enough to generate a rapidly varying structure into the three-body amplitude. Further, we may determine whether that structure is kinematical in origin or whether it corresponds to a pole in the three-body amplitude generated by the two-body virtual state pole.

Following this program, we employ the same proton-molecule Yamaguchi $s$-wave separable potential as in Ref. 2. This potential has a bound state at $E_{p c}=-3.5 \mathrm{eV}$ and a rms vibrational amplitude of $0.23 \AA$. We consider a number of arti ficial $n-p s$-wave Yamaguchi separable interactions, each with an effective range of $2.91 \mathrm{fm}$ and with scattering lengths of $a_{n p}^{(1)}=-1.275 \times 10^{1}, a_{n p}^{(2)}$ $=-1.275 \times 10^{2}, a_{n p}^{(3)}=-1.275 \times 10^{3}, a_{n p}^{(4)}=-1.275$ $\times 10^{4}, a_{n p}^{(5)}=-1.275 \times 10^{5}$, and $a_{n p}^{(6)}=-1.275 \times 10^{6}$ $\mathrm{fm}$. The value of $a_{n p}^{(1)}$ is chosen so that if the actual singlet and triplet potentials were replaced by an effective $n-p$ potential with $a_{n p}=-12.75 \mathrm{fm}$, the 


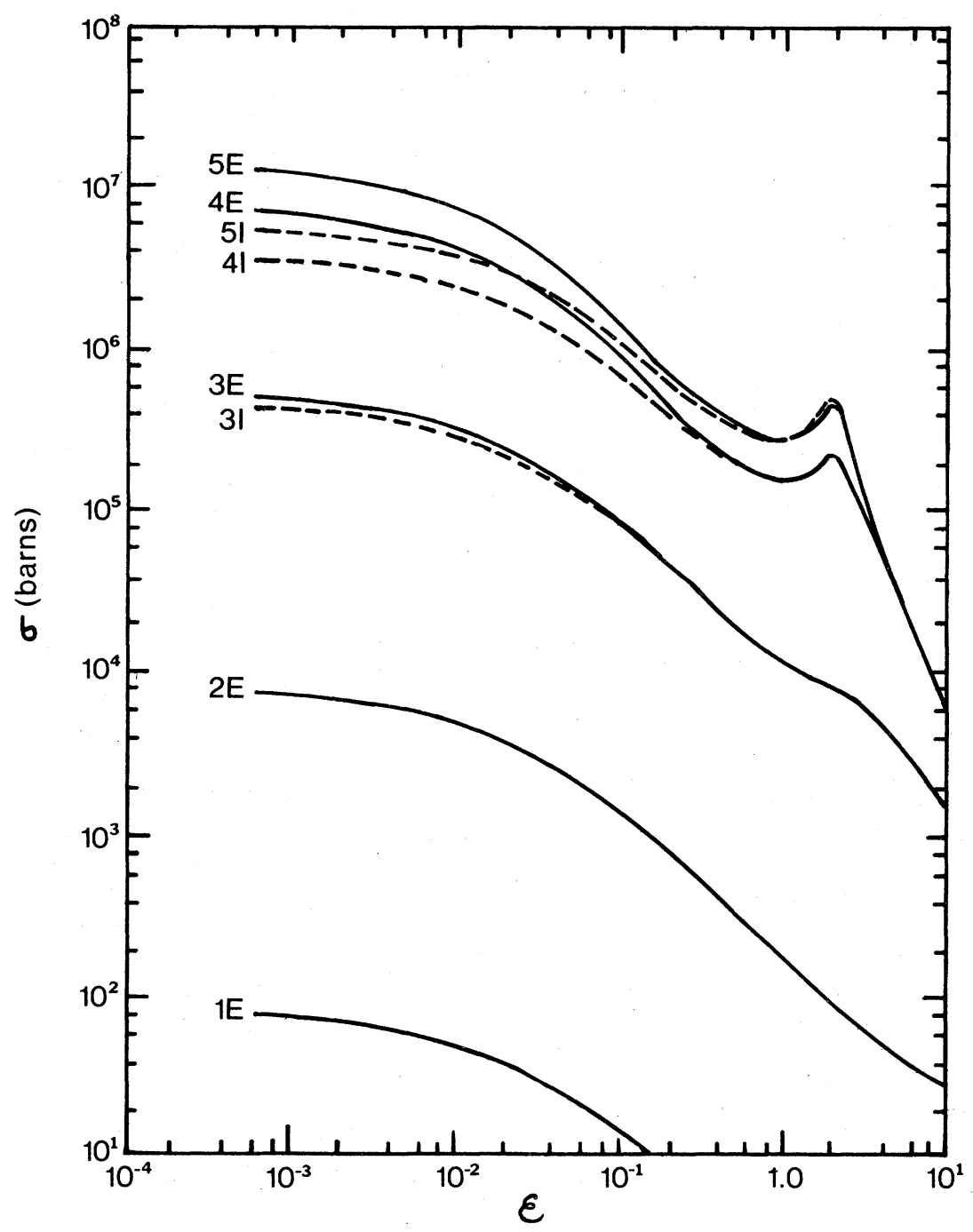

FIG. 1. Elastic total cross sections as functions of the initial neutron energy as defined in Eq. (1) for the six artificial $n-p$ potentials with effective range $r_{n p}=2.91 \mathrm{fm}$. and zero energy scattering lengths $a_{n p}^{(j)}=-1.275 \times 10^{j} \mathrm{fm}$ with 1 $\leq j \leq 6$. The solid curves labeled by $(E)$ are the exact Faddeev cross sections and the dashed curves labeled by $(I)$ are the impulse approximation cross sections. The cross sections for potential (6) are not shown, the results being larger than the potential (5) cross sections by $7.8 \%$ at $\mathcal{E}=6.0 \times 10^{-4}$, by $5.3 \%$ at $\mathcal{E}=1.5$, and by $0.6 \%$ at $\mathcal{E}=3.0$.

observed zero energy free cross section would result. Using these potentials in Eqs. (2.6)-(2.8) and (2.14)-(2.16) of Ref. 2, we have calculated the impulse total elastic cross section and the exact total elastic cross section as functions of initial neutron energy $e_{n}$. The exact cross sections were obtained by adding the $J \geqslant 6$ partial wave impulse cross sections to the $0 \leqslant J \leqslant 5$ cross sections obtained by inversion of the partial wave Faddeev equations. These cross sections are shown in Fig. 1 and the corresponding cross sections for the first six partial waves for $n-p$ potential (5) are shown in Fig. 2.

An examination of these figures shows the fol- lowing: First, as expected, the impulse approximation constitutes an accurate approximation to the exact scattering at high energies, in high partial waves, and in the so-called "quasiclassical binding" case where the proton-molecule force is slowly varying over the $n p$ interaction volume. ${ }^{3}$ The most interesting feature of the curves is that as $a_{n p} \rightarrow-\infty$ an enhancement builds up in the elastic three-body total cross section at an initial neutron energy of

$$
\mathcal{E} \equiv \frac{e_{n}}{\left|E_{p c}\right|}=2.0
$$

This enhancement may be explained kinematically 


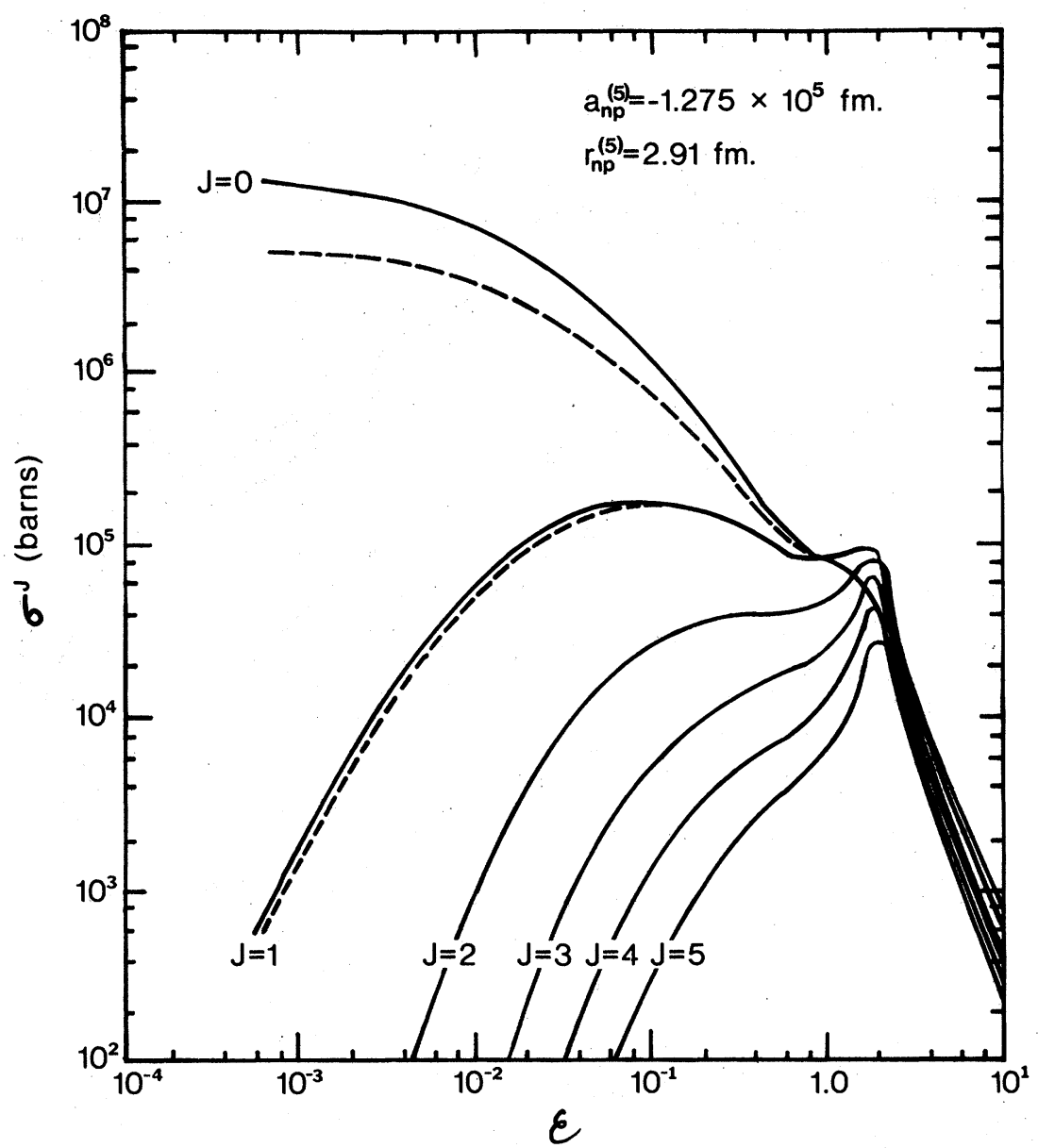

FIG. 2. Elastic partial wave cross sections as functions of the initial neutron energy as defined in Eq. (1) for the first six partial waves and for potential (5) with $r_{n p}=2.91 \mathrm{fm}$ and $a_{n p}^{(5)}=-1.275 \times 10^{5} \mathrm{fm}$. The solid curves are the exact Faddeev cross sections and the dashed curves are the impulse approximation cross sections.

as follows. For $\mathcal{E} \gtrsim 1.0$ the impulse approximation adequately describes the exact scattering and is given by the expression

$$
\begin{aligned}
T_{\mathrm{imp}}^{\mathrm{el}}\left(\overrightarrow{\mathrm{q}}_{n}^{\prime}, \overrightarrow{\mathrm{q}}_{n}\right)=\int \frac{d^{3} q_{c}^{\prime \prime}}{(2 \pi)^{3}} \phi_{p c}^{*}\left(\overrightarrow{\mathrm{q}}_{n}^{\prime}+\overrightarrow{\mathrm{q}}_{c}^{\prime \prime}\right) \phi_{p c}\left(\overrightarrow{\mathrm{q}}_{n}+\overrightarrow{\mathrm{q}}_{c}^{\prime \prime}\right) \\
\quad \times\left\langle\overrightarrow{\mathrm{q}}_{n}^{\prime}+\frac{1}{2} \overrightarrow{\mathrm{q}}_{c}^{\prime \prime}\right| t_{n p}\left(\frac{q_{n}^{2}}{2 m_{n}}-\left|E_{p c}\right|\right. \\
\left.-\frac{q_{c}^{\prime \prime 2}}{4 m_{n}}\right)\left|\overrightarrow{\mathrm{q}}_{n}+\frac{1}{2} \overrightarrow{\mathrm{q}}_{c}^{\prime \prime}\right\rangle,
\end{aligned}
$$

where $\phi_{p c}$ is the proton-core wave function. In Eq. (2) the wave functions peak strongly at

$$
\left|\overrightarrow{\mathrm{q}}_{n}^{\prime}+\overrightarrow{\mathrm{q}}_{c}^{\prime \prime}\right|=\left|\overrightarrow{\mathrm{q}}_{n}+\overrightarrow{\mathrm{q}}_{c}^{\prime \prime}\right|=0 .
$$

The presence of the virtual state produces a peaking in $t_{n p}$ about $\boldsymbol{E}=0$. This $\boldsymbol{E}=0$ peaking condition in Eq. (2) is

$$
0=\frac{q_{n}^{2}}{2 m_{n}}-\left|E_{p c}\right|-\frac{q_{c}^{\prime 2}}{4 m_{n}}
$$

These two equations are simultaneously satisfied for

$$
e_{n} \equiv \frac{q_{n}^{2}}{2 m_{n}}=2\left|E_{p c}\right|
$$

which is the position of the enhancement in Figs. 1 and 2. Similarly, when $\left|a_{n p}\right| \ll a_{p c}$ where $a_{p c}$ is the proton-molecule scattering length, the peaking in $t_{n p}(E)$ is negligible leading to a substantial broadening and flattening of the corresponding three-body enhancement as seen in Fig. 1. It should be noted that this kinematical argument may also be extended to the case of resonances since these also produce a sharp peaking in the projectile-target $t$ matrix.

In Fig. 1, the asymptotic limits of the cross sections for $a_{n p} \rightarrow-\infty$ and $a_{n p} \rightarrow 0$ are seen to be well behaved, the cross section becoming indepen- 
dent of $a_{n p}$ as $a_{n p} \rightarrow-\infty$ and scaling as ${a_{n p}}^{2}$ as $a_{n p} \rightarrow 0$. For $a_{n p} \rightarrow 0$, the $n p t$-matrix element is very slowly varying about the on shell zero energy value [Eq. (13.5) of Ref. 2],

$$
\lim _{a_{n p} \rightarrow 0}\left\langle\overrightarrow{\mathrm{k}}^{\prime}\left|t_{n p}(E)\right| \overrightarrow{\mathrm{k}}\right\rangle=4 \pi a_{n p} / m_{n} \text {. }
$$

Substituting this into Eq. (2) and recalling that the $a_{n p} \rightarrow 0$ limit is the "quasiclassical binding" case mentioned above, we obtain

$$
\lim _{a_{n p} \rightarrow 0}\left(\frac{d \sigma}{d \Omega}\right)^{\mathrm{el}}\left(\overrightarrow{\mathrm{q}}_{n}^{\prime}, \overrightarrow{\mathrm{q}}_{n}\right) \approx 4 a_{n p}^{2} F_{p c}^{2}\left(\left|\overrightarrow{\mathrm{q}}_{n}^{\prime}-\overrightarrow{\mathrm{q}}_{n}\right|\right)
$$

where $F_{p c}$ is the proton-molecule form factor. For $a_{n \phi} \rightarrow-\infty$, the $\tau$ function, which for the $s-$ wave separable potential is given by

$$
\left\langle\overrightarrow{\mathrm{k}}^{\prime}\left|t_{n p}(E)\right| \overrightarrow{\mathrm{k}}\right\rangle=g_{n p}\left(k^{\prime}\right) \tau_{n p}(E) g_{n p}(k),
$$

becomes very strongly peaked about $E=0$. To determine the asymptotic behavior in the context of the impulse approximation we perform the angular integrations in Eq. (2), and obtain approxi mately (exactly for constant $g$ and Gaussian $\phi$ )

$$
\begin{array}{r}
\lim _{a_{n p} \rightarrow-\infty} T_{i m p}^{\mathrm{el}}\left(\overrightarrow{\mathrm{q}}_{n}^{\prime}, \overrightarrow{\mathrm{q}}_{n}\right) \propto \int_{-\infty}^{E_{3-\text { body }}>0} d E_{n p}^{\prime \prime} \tau_{n p}\left(E_{n p}^{\prime \prime}\right) \\
H\left(E_{n p}^{\prime \prime}, q_{n}, \cos \theta_{\alpha_{n}^{\prime} a_{n}}\right),
\end{array}
$$

where $H$ represents the result of the angular integration. For the Yamaguchi $s$-wave virtual state $t$ matrix, the peaking about $E=0$ is described by

$$
\tau_{n p}(0) \propto\left|a_{n p}\right|
$$

and

$$
\lim _{a_{n p} \rightarrow-\infty} \int_{A}^{B} \tau_{n p}(E) d E=I_{n p},
$$

where $A \ll 0 \ll B$ and where $I_{n p}$ is a finite nonzero constant. With these relations, Eq. (9) finally becomes

$$
\lim _{a_{n p \rightarrow-\infty}} T_{\mathrm{imp}}^{\mathrm{el}}\left(\overrightarrow{\mathrm{q}}_{n}^{\prime}, \overrightarrow{\mathrm{q}}_{n}\right) \propto I_{n p} H\left(0, q_{n}, \cos \theta_{a_{n}^{\prime} \alpha_{n}}\right),
$$

independent of the scattering length $a_{n p}$. The width of the enhancement in the three-body cross section in this limit is determined only by the width of the momentum distribution of the protoncore wave function.

The final feature of the three-body elastic cross sections is that, as in Fig. 2, the enhancement appears in many partial waves. This was verified by examining the phase of each partial wave amplitude. Just as the two-body virtual state phase shift rises rapidly from zero to $\delta \leqslant 90^{\circ}$ near zero two-body energy, so too did the phases of the partial wave three-body amplitudes rapidly rise to $\delta_{J} \leqslant 90^{\circ}$ near $\mathcal{E}=2.0$. In the two-body case when a partial wave analysis is performed on the cor- responding $t$ matrix, it is an extremely unlikely circumstance that two or more of the independent partial wave amplitudes resonate simultaneously. However, since the three-body angular momentum is obtained by a successive coupling of two-body angular momenta, a single resonant two-body partial wave may contribute to all total angular momentum states. This is a result particular to an $N \geqslant 3$-body scattering situation; i.e., when an enhancement is observed simultaneously in many projectile-target partial wave channels, it is indicative that the target is a composite system rather than a particle which is in some sense elementary ${ }^{4}$ although the imposition of additional symmetries such as parity may restrict the range of allowable resonant channels. ${ }^{5,6}$ It may be argued that our three-body enhancements arise from spurious Peierls singularities since these spurious enhancements also appear in each partial wave channel. ${ }^{7}$ However, our formalism does include off-shell intermediate state propagation ${ }^{8,9}$ thus removing the Peierls singularities to their correct sheet, far from the physical region.

Aside from the kinematical explanation of the enhancement, one may ask whether the enhancement is the signature of a pole in the three-body amplitude generated by the virtual state pole in the $n p$ subsystem. ${ }^{10}$ Such poles have been shown by Brayshaw and Peierls ${ }^{4}$ to exist for a one dimensional system of three equal mass particles. Numerical searches ${ }^{11-15}$ have been made for such poles with a questionable degree of success for more complicated three-dimensional systems with subsystem channel resonances. For these more complex systems the theory of Brayshaw and Peierls is less definite as to the existence of three-body poles although they do suggest likely places where such generated poles might be found. Since our enhancements are strongly present in the impulse approximation, we calculated the im pulse amplitude off-shell in the three-body parametric energy and performed a numerical search for three-body poles below the breakup threshold. None were found. Similarly, a numerical search was performed below the breakup threshold on the inverse Fredholm determinant for the exact Faddeev amplitude. Again no pole structure was encountered. From these null results we conclude that our enhancements are merely kinematical in origin as explained above and do not reflect the presence of a three-body pole generated by the two-body subchannel virtual state pole.

As a historical note, it is the high energy of the $n p$ virtual state with respect to molecular binding energies which causes the $n p t$ matrix to be quite flat allowing Fermi's pseudopotential method to well describe the system and predict the wellknown factor of 4 ratio between the zero energy 
neutron-bound proton cross section and the zero energy free $n p$ cross section as in Eq. (7). If the $n p$ virtual state energy had been four orders of magnitude lower $(E \leq 5 \mathrm{eV})$, the $n p t$ matrix would have been sufficiently rapidly varying about $E=0$ so that the ratio of the zero-energy bound-to-free cross sections would not have been as in Fig. 1 an even and easily interpretable factor of 4.0 . In . the Reid hard core model ${ }^{16}$ of the $n-p$ potential, the singlet-s $n-p$ virtual state energy decreases by four orders of magnitude when the non-one-pionexchange portion of the potential is increased from its physical value by $5.20 \%$. Likewise, it takes only a $5.25 \%$ increase in the strength of the non-OPE potential to bind the singlet-s system. Therefore although the physical $n-p$ potential gives rise to Fermi's factor of 4.0 , only a minor increase of the short range portion of the potential would have given rise to a very different neutron-bound proton cross section whose interpretation would have required a much more sophisticated (i.e., Faddeev) theory than the pseudopotential theory.

\section{ACKNOWLEDGMENTS}

The author would like to thank Professor L. L. Foldy for many helpful suggestions during the course of this work. Most of the numerical portion of this study was carried out while the author was associated with Case Western Reserve University. This work was supported, in part, by the National Science Foundation under Grant PHY76-00299.
*Permanent address.

${ }^{1}$ E. Fermi, Ricerca Sci. 7, 13 (1936). [Translation: Enrico Fermi: Collected Papers, Vol. 1 (University of Chicago Press, Chicago, 1962)].

${ }^{2} \mathrm{~J}$. A. Lock, Nucl. Phys. A298, 253 (1978).

${ }^{3}$ M. L. Goldberger and K. M. Watson, Collision Theory (Wiley, New York, 1964) Sec. 11.1.

${ }^{4}$ D. D. Brayshaw and R. F. Peierls, Phys. Rev. 177, 2539 (1968).

${ }^{5}$ D. D. Brayshaw, Phys. Rev. Lett. 37, 1329 (1976).

${ }^{6} \mathrm{~J}$. A. Lock, Nucl. Phys. A271, 458 (1976).

${ }^{7}$ M. Nauenberg and A. Pais, Phys. Rev. Lett. 8,82 (1962).

${ }^{8}$ I. J. R. Aitchison and C. Kacser, Phys. Rev. 173, 1700 (1968).
${ }^{9}$ I. J. R. Aitchison and R. J. A. Golding, Phys. Lett. 59B, 288 (1975).

${ }^{10}$ R. C. Hwa, Phys. Rev. 130, 2580 (1963).

${ }^{11} \mathrm{~J}$. -L. Basdevant and R. E. Kreps, Phys. Rev. 141, 1398 (1966); 141, 1404 (1966); 141, 1409 (1966).

${ }^{12} \mathrm{~J} .-\mathrm{L}$. Basdevant and R. L. Omnès, Phys. Rev. Lett. 17, 775 (1966)

${ }^{13}$ G. Mennessier, J. $-Y$. Pasquier, and R. Pasquier, Phys. Rev. D 6, 1351 (1972).

${ }^{14}$ I. J. R. Aitchison and D. Krúpa, Nucl. Phys. A182, 449 (1972).

${ }^{15}$ A. M. Badalyan and Yu. A. Simonov, Yad. Fiz. 21, 890 (1975) [Sov. J. Nucl. Phys. 21, 458 (1975)].

${ }^{16}$ R. V. Reid, Ann. Phys. (N. Y.) $\underline{50}, 411$ (1968). 